\title{
AN02/DNP-hapten unbinding forces studied by molecular dynamics atomic force microscopy simulations
}

\author{
Berthold Heymann, Helmut Grubmüller* \\ Max-Planck-Institute for Biophysical Chemistry, Theoretical Molecular Biophysics Group, Am Faßberg 11, 37077 Göttingen, Germany
}

Received 14 January 1999; in final form 8 February 1999

\begin{abstract}
Unbinding forces of a spin-labeled dinitrophenyl (DNP) hapten from the monoclonal antibody AN02 $\mathrm{F}_{\mathrm{ab}}$ fragment have been studied by molecular dynamics atomic force microscopy (AFM) simulations. In our nanosecond simulations, unbinding was enforced by pulling the hapten molecule out of the binding pocket. From the simulations unbinding forces of the complex have been determined as a function of pulling velocity. Considering activated unbinding and frictional forces, we used a simple model to extrapolate the unbinding forces to the millisecond timescale of single molecule AFM unbinding experiments. For such experiments the simulations suggest an unbinding force of $60 \pm 30$ pN. (C) 1999 Elsevier Science B.V. All rights reserved.
\end{abstract}

\section{Introduction}

Molecular recognition is essential for many biochemical processes and is realized by highly specific binding of ligands to their receptors. In the immune system antibodies play a crucial role by recognizing and specifically binding their antigens. A huge amount of calorimetric, spectroscopic, kinetic, and structural data on antibody/antigen systems has been accumulated (cf. Ref. [1] and references therein), and antibodies are widely used in biotechnology, pharmacology [2], and as catalysts [3]. To advance our understanding of the antigen binding mechanism, detailed insight into its molecular dynamics at the atomic level is essential.

\footnotetext{
* Corresponding author. Fax: +49 551201 1089; e-mail: hgrubmu@gwdg.de
}

Recent advances in single molecule atomic force microscopy (AFM) and other force microscopy techniques allow us to study mechanical properties of individual molecules like the binding forces of protein-ligand complexes $[4,5]$, the enforced unfolding of proteins [6-8], or the stiffness of other polymers like DNA [9] or polysaccharides [10]. Also, and motivating the present work, single molecule AFM studies on a number of antibody/antigen complexes have been carried out [11-14], or are under way.

Computer simulations of such AFM experiments [10,15-19] by means of molecular dynamics (MD) simulations [20] can provide models that explain the measured forces in terms of molecular structures, unbinding reaction pathways, and interatomic interactions $[10,15]$. They thereby complement single molecular AFM experiments with microscopic interpretations. Such simulations can and have to be validated by computing unbinding forces from the 
simulations and comparing these with experiment [15]. However, such comparison is complicated by the fact that AFM experiments are typically carried out at a millisecond timescale or slower, whereas MD simulations are currently limited to nanoseconds. Since the unbinding force generally depends on how fast unbinding is enforced, care has to be taken when relating simulations to experiment $[15,17,18]$.

Here we report the computation of unbinding forces from a series of extended MD AFM simulations of a solvated antibody (AN02) $F_{a b}$ fragment complexed with a spin-labeled dinitrophenyl hapten (DNP-SL), the structure of which has been solved recently [21], and for which single molecule AFM data will likely be available soon. Hence, comparison of that data with our computed unbinding forces could validate their microscopic interpretation that can be extracted from our simulations. This Letter will mainly focus at the unbinding forces; a detailed structural interpretation will be given elsewhere. Additionally, we want to quantify the typical scatter of unbinding forces, which has already been observed in other systems [5,15], and which we assume to reflect a structural heterogeneity in the unbinding pathways of the ligand out of the binding pocket.

To relate computed unbinding forces to experimental ones, we will consider frictional forces and activated barrier crossings as the two main causes for the timescale dependence of unbinding forces. These have been discussed within the general framework of stochastic processes and rate theory [17,22]. Unfortunately, analytical results that would allow extrapolation of computed unbinding forces to the experimental timescale could only be obtained for simplified binding interaction potentials [17]. In these models, friction was assumed to be linearly related to pulling velocity according to Langevin theory [17], whereas activated processes, if described by Kramers' theory, lead to a logarithmic dependence of the unbinding force on the loading rate. As already suggested in Ref. [15] and discussed in Ref. [17], frictional effects are expected to contribute significantly to the unbinding force of ligand/receptor complexes for pulling velocities above about $1 \mathrm{~m} / \mathrm{s}$ ('friction regime'), whereas activated processes dominate for slower velocities ('activated regime'), for which frictional forces become negligible.
Inspired by these results, we will combine the two effects and extrapolate our computed unbinding forces to the AFM timescale of milliseconds. Since the extrapolation, if based solely on the simulations, is expected to be unstable, we will use the spontaneous dissociation rate for the AN02/DNP-SL complex as additional information.

In Section 2 we first describe the AFM simulations that have been carried out; subsequently the theory underlying our extrapolation is described.

\section{Methods}

\subsection{Simulations}

As a starting point for the simulations the X-ray structure of the AN02 $\mathrm{F}_{\mathrm{ab}}$ fragment complexed with the DNP-SL ligand [21] was taken from the Brookhaven Protein Data Bank [23], entry 1baf. All molecular dynamics simulations were performed with the parallel MD program EGO [24], which uses the CHARMM force field [25]. EGO allows efficient computation of Coulomb forces using the 'fast multiple time step structure adapted multipole method' [26], so that no artificial truncation of these forces had to be applied. For the hapten, partial charges were calculated using the program UNICHEM [27]; the force constants were taken from Ref. [25]. All simulations were carried out with an integration step size of $10^{-15} \mathrm{~s}$. The length of chemical bonds involving hydrogen atoms were fixed [28], and nonpolar hydrogen atoms were treated through compound atoms [25]. No explicit term for the hydrogen bond energy was included within the force field.

A model for the solvated protein-ligand complex was created by surrounding its X-ray structure with a droplet containing 13461 TIP3 [29] water molecules and 75 ions $\left(\mathrm{Na}^{+}, \mathrm{Cl}^{-}\right)$at physiological concentration using the program SOLVATE [30]. The ions were placed according to a Debye-Hückel distribution governed by the protein charges. To keep the number of solvent molecules in the system as small as possible, a non-spherical solvent volume was chosen. The solvent surface was defined such that the minimum distance between protein surface and the solvent surface was not less than $20 \AA$ near the binding pocket region and not less than $12 \AA$ else- 
where. The obtained simulation system comprised a total of 44571 atoms.

To counterbalance surface tension and to prevent evaporation of water molecules, all surface water molecules were subjected to 'deformable boundary forces' [31] (approximated by a quartic polynomial as described in Refs. [32,33]). Additionally, all water molecules at the surface of the droplet were coupled to a heat bath of $300 \mathrm{~K}$ through stochastic forces obeying the fluctuation-dissipation theorem using a coupling constant of $\beta=10 \mathrm{ps}^{-1}$ as described in Ref. [15]. All other atoms were weakly coupled to a heat bath $\left(\beta=1 \mathrm{ps}^{-1}\right)$ via velocity rescaling as described in Ref. [20]. After minimization, the system was equilibrated for 1300 ps. During equilibration, the stability of the simulation system was monitored via the root mean square (rms) deviation from the X-ray structure considering all heavy backbone atoms (1) of the complete protein-ligand complex, (2) of the variable and (3) constant domain regions as well as (4) of the binding pocket, respectively. As the binding pocket we defined all residues that interact with the hapten molecule during the unbinding process and parts of the hypervariable loops that are located next to the hapten molecule; these comprised light chain residues $30-35,47-52$, and $86-99$, as well as heavy chain residues $30-36,48-55$, and 97-105, respectively.

For the subsequent MD AFM simulations we proceeded as described in Ref. [15] and sketched in Fig. 1. Resembling the pulling forces exerted in the AFM experiment by the cantilever, the spin-labeled oxygen atom ' $\mathrm{O} 2$ ' of the hapten was subjected to a spring potential $V_{\text {spring }}$,

$$
V_{\text {spring }}=\frac{1}{2} k_{\text {cant }}\left[z_{\mathrm{O} 2}(t)-z_{\text {cant }}(t)\right]^{2} \text {, }
$$

acting on the $z$-coordinate $z_{\mathrm{O} 2}$ of atom $\mathrm{O} 2$. Here, $k_{\text {cant }}=280 \mathrm{pN} / \AA$ is the spring constant and $z_{\text {cant }}(t)$ is the 'cantilever' position. In Fig. 1, that pulling potential is symbolized by a 'spring'. Note that $V_{\text {spring }}$, as defined above, does not restrain sideward motions of the $\mathrm{O} 2$ atom, i.e., perpendicular to the $z$-direction, since such motions are also essentially unconstrained in the experiments.

At the beginning of each MD AFM simulation the minimum position, $z_{\text {cant }}(0)$, of $V_{\text {spring }}$ was placed at

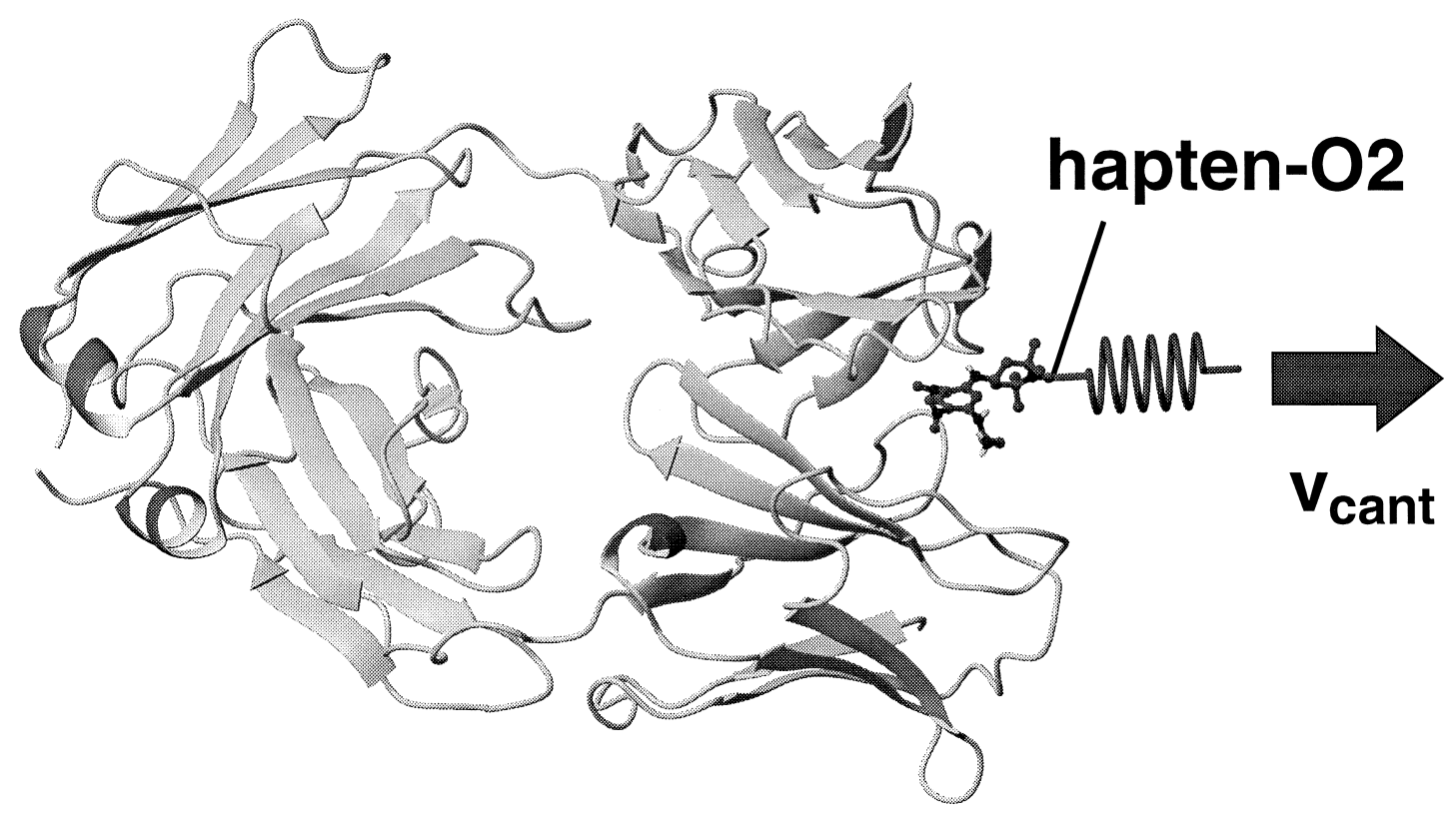

Fig. 1. Model for the simulation of single molecule atomic force microscopy rupture experiments on the AN02-hapten complex. The hapten (ball-and-stick model) was pulled out of the binding pocket of AN02 (shown as a ribbon model) through a harmonic potential (symbolized by a spring) that was applied to the hapten oxygen atom $\mathrm{O} 2$. This pulling potential was moved with constant velocity $v_{\text {cant }}$ (arrow), while the center of mass of the AN02 was kept in place. 
the $\mathrm{O} 2$ position, $z_{\mathrm{O} 2}(0)$. In the course of each simulation, $V_{\text {spring }}$ was moved in pulling direction (arrow), $z_{\text {cant }}(t)=z_{\text {cant }}(0)+v_{\text {cant }} t$, with constant pulling velocity $v_{\text {cant }}$.

To avoid larger sideward drift of the hapten, atom $\mathrm{O} 2$ was additionally subjected to a weak harmonic potential (spring constant: $14 \mathrm{pN} / \AA$ ) perpendicular to the pulling direction. Finally, the center of mass of the protein (with hapten excluded) was kept in place by a stiff harmonic potential (force constant: 2800 $\mathrm{pN} / \AA$ ), thus allowing the protein to adapt to the enforced hapten unbinding, e.g., by rotations or intramolecular conformational motions in close resemblance to typical AFM experiments.

During the enforced unbinding, the pulling force $F_{\text {pull }}(t)=k_{\text {cant }}\left[z_{\text {cant }}(t)-z_{\mathrm{O} 2}(t)\right]$ acting on the oxygen atom was calculated and recorded every 100 fs, yielding a 'force profile' for each MD run as a function of cantilever position $z_{\text {cant }}(t)$. From each AFM simulation run, an unbinding force was derived as the maximum of the respective force profile, and the position of atom $\mathrm{O} 2$ at that maximum was recorded to obtain an 'unbinding length'. As described in Refs. $[15,18]$, care has to be taken here to eliminate by proper smoothing thermal high frequency force fluctuations which do not pertain to the binding forces of interest, and which would cause an overestimate of the maximum pulling force. Motivated by the fact that the binding forces mainly result from local interactions - like van der Waals contacts or hydrogen bonds - with individual rupture lengths of typically $0.3 \AA$ or larger [15], we chose a Gaussian filter with $0.3 \AA$ half width for that purpose. To quantify the uncertainty introduced by the particular choice of the smoothing filter, error bars were determined by considering force profiles filtered with 0.1 and $0.5 \AA$ half widths, respectively.

A total of 39 MD AFM simulations was carried out. To compute the unbinding force as a function of pulling velocity we chose pulling velocities in the range between 0.1 and $50 \mathrm{~m} / \mathrm{s}$ for these MD AFM runs, which required simulations of 30-7000 ps duration to complete the unbinding process.

\subsection{Theory}

To extrapolate the computed unbinding forces from the nanosecond MD timescale to the millisecond AFM timescale we combined the observation that for slow pulling velocities the unbinding forces increase logarithmically with pulling force $[6,34]$ with the assumption that for enforced fast unbinding, Stokes' friction, $F_{\text {friction }}=\gamma v_{\text {cant }}$, with friction coefficient $\gamma$ significantly contributes to the unbinding force. That assumption is supported by a linear relationship between unbinding force and pulling velocity observed in previous simulations [15] as well as by the simulations presented here (cf. Section 3 ); additional support comes from the analysis of a one-dimensional memory-free diffusion model for the unbinding process [17]. In the latter model, three regimes were distinguished: a drift regime for fast pulling velocities, an activated regime for slow pulling velocities, and a diffusion regime, which connects drift and activated regime. Accordingly, we approximate the total unbinding force, $F_{\text {unbind }}\left(v_{\text {cant }}\right)$, with a sum

$F_{\text {unbind }}\left(v_{\text {cant }}\right)=F_{\text {friction }}\left(v_{\text {cant }}\right)+F_{\text {act }}\left(v_{\text {cant }}\right)$

of the above-mentioned Stokes' friction $F_{\text {friction }}\left(v_{\text {cant }}\right)$ and a force contribution $F_{\text {act }}\left(v_{\text {cant }}\right)$ governed by activated crossing of unbinding free energy barriers.

To describe the latter as a function of pulling velocity, we consider an ensemble of unbinding processes for given pulling velocity $v_{\text {cant }}$. Denoting the instance of maximal flux across the unbinding energy barrier as the rupture point, we focus at a time interval $\Delta T_{\text {trans }}$, within which most of the unbinding events occur. We assume that for the given pulling velocity $v_{\text {cant }}$ the applied pulling force $F_{\text {pull }}(t)$ does not vary significantly within $\Delta T_{\text {trans }}$ and refer to its average value as $F_{\text {act }}$. Within that approximation the unbinding process can be described as an activated crossing of an unbinding free energy barrier $\Delta G^{\ddagger}$, which, due to the pulling force $F_{\text {act }}$, is reduced to $\Delta G_{v}^{\ddagger}$,

$\Delta G_{v}^{\ddagger}=\Delta G^{\ddagger}-L \cdot F_{\text {act }}$.

Here, $L$ denotes the average unbinding length, defined as the average displacement of the hapten oxygen atom $\mathrm{O} 2$ from its initial position at the rupture point. In Eq. (3), and also subsequently, the index $v$ refers to non-zero pulling velocity, i.e., enforced unbinding, whereas the index 0 will refer to spontaneous dissociation of the AN02/DNP complex.

To relate $F_{\text {act }}$ to $v_{\text {cant }}$, we express the average enforced dissociation rate $k_{v}$ of the complex within 
the time interval $\Delta T_{\text {trans }}$ as a function of $\Delta G_{v}^{\ddagger}$ using the Kramers approximation,

$k_{v}=\omega_{0} \exp \left(-\frac{\Delta G_{v}^{\ddagger}}{k_{\mathrm{B}} T}\right)$.

Here, $\omega_{0}$ is the Kramers prefactor that describes the fluctuations of the system within the bound state, $k_{\mathrm{B}}$ is the Boltzmann constant, and $T$ the temperature. Note that now $k_{v}$ can also be approximated by $k_{v} \approx 1 / \Delta T_{\text {trans }}$, since, by definition, essentially all unbinding events occur within $\Delta T_{\text {trans }}$. With the scatter width $\Delta L$ of the individual unbinding lengths, $\Delta L:=v_{\text {cant }} \Delta T_{\text {trans }}$, the enforced unbinding rate reads

$k_{v}=\frac{v_{\text {cant }}}{\Delta L}$.

We use the Kramers approximation also for spontaneous dissociation,

$k_{0}=\omega_{0} \exp \left(-\frac{\Delta G^{\ddagger}}{k_{\mathrm{B}} T}\right)$,

(with spontaneous dissociation rate $k_{0}$ ) and assume that the Kramers prefactor $\omega_{0}$ used in Eq. (6) does not differ significantly from the one used in Eq. (4). That assumption is supported by the observation that in our simulations the amplitude of the fluctuations of the oxygen atom $\mathrm{O} 2$ is changed by the pulling potential by less than a factor of two when compared to the equilibrium simulation (data not shown). The same holds for the center of mass of the hapten. Eqs. (3)-(6) yield the desired result,

$F_{\text {act }}\left(v_{\text {cant }}\right)=\frac{k_{\mathrm{B}} T}{L} \ln \frac{v_{\text {cant }}}{k_{0} \Delta L}$.

Eq. (7) expresses the logarithmic variation of the unbinding force with pulling velocity within the activated regime and properly considers the spontaneous dissociation rate.

By fitting $F_{\text {unbind }}\left(v_{\text {cant }}\right)$, as defined by Eqs. (2) and (7) to the unbinding forces derived from the AFM simulations one can now estimate unbinding forces at experimental timescales. As adjustable parameters we used $L, \Delta L$, and the friction coefficient $\gamma$. The only quantity which cannot reliably be ex- tracted from the simulations is the spontaneous dissociation rate, $k_{0}$, which we therefore took from experimental data. Unfortunately, to our knowledge $k_{0}$ has not yet been measured for AN02/DNP-SL, so its value had to be estimated from the dissociation rates that have been measured for the dinitrophenylglycine $\left(k_{0} \approx 100 / \mathrm{s}\right)$ and dinitrophenyldiglycine $\left(k_{0}\right.$ $\approx 130 / \mathrm{s})$ analogues [35], which exhibit slightly smaller binding constants than AN02/DNP-SL. Accordingly, we assumed $k_{0}=110 \pm 70 /$ s for AN02/ DNP-SL.

To separate the uncertainty of our predicted unbinding force that is due to the inaccurate value of $k_{0}$ from the uncertainty caused by the scatter of the calculated forces observed in the simulations, we express $F_{\text {act }}$ in terms of the (logarithmic) acceleration $\alpha_{v}$,

$\alpha_{v}=\ln \frac{k_{v}}{k_{0}}$.

To rewrite Eq. (7) in a more convenient form for the activated regime $\left(v_{\text {cant }}<1 \mathrm{~m} / \mathrm{s}\right)$, we now assume that there is a $v_{\text {cant }}^{*} \approx 1 \mathrm{~m} / \mathrm{s}$ for which the unbinding force derived from the fit of Eqs. (2) and (7) to the simulation data as well as to $k_{0}$ is essentially independent of the particular choice of $k_{0}$ (this assumption will be verified in Section 3). Now Eq. (7) can be cast in the form

$F_{\text {unbind }} \approx F_{\text {act }}=F^{*} \frac{\alpha_{v}^{\exp }}{\alpha_{v}^{*}}$,

where the superscripts ' $*$ ' and 'exp' refer to the chosen $v_{\text {cant }}^{*}$ and to the experimental timescale for which the unbinding force is to be estimated, respectively.

Eq. (9) emphasizes that the unbinding force scales linearly with the logarithmic acceleration factor, and the coefficient of proportionality, $F^{*} / \alpha_{v}^{*}$, can be readily derived from the series of AFM MD simulations.

\section{Results}

During the 1300 ps equilibration phase, the solvated antibody-hapten complex exhibited relaxation motions for more than 1100 ps. In particular, slight 
tilt motions of the variable region domain of the $\mathrm{F}_{\mathrm{ab}}$ fragment (cf. Fig. 1, right) with respect to the constant region domain (left) caused a relatively large rms deviation from the X-ray structure. This deviation built up monotonously from a value of $2.2 \AA$ at 100 ps to a maximum value of $3.2 \AA$ at 1100 ps, at which it stabilized. The rms deviations for the variable region domain $(2.3 \AA)$ and for the constant region domain $(2.7 \AA)$, respectively, were significantly smaller and leveled off already after 900 ps. The relevant binding pocket stabilized even earlier after 500 ps with a maximum rms deviation of only $1.6 \AA$. From the latter value we assumed that the system, and in particular the binding pocket, was sufficiently equilibrated after 1300 ps.

Fig. 2 displays a typical force profile obtained from one of the subsequent AFM MD simulation runs with pulling velocity $v_{\text {cant }}=5 \mathrm{~m} / \mathrm{s}$. From the global maximum of the force profile an unbinding force (see Section 2) has been obtained as indicated in the figure. The error bar measures the uncertainty introduced by filtering the thermal high frequency fluctuations in the force profile using Gaussian filters

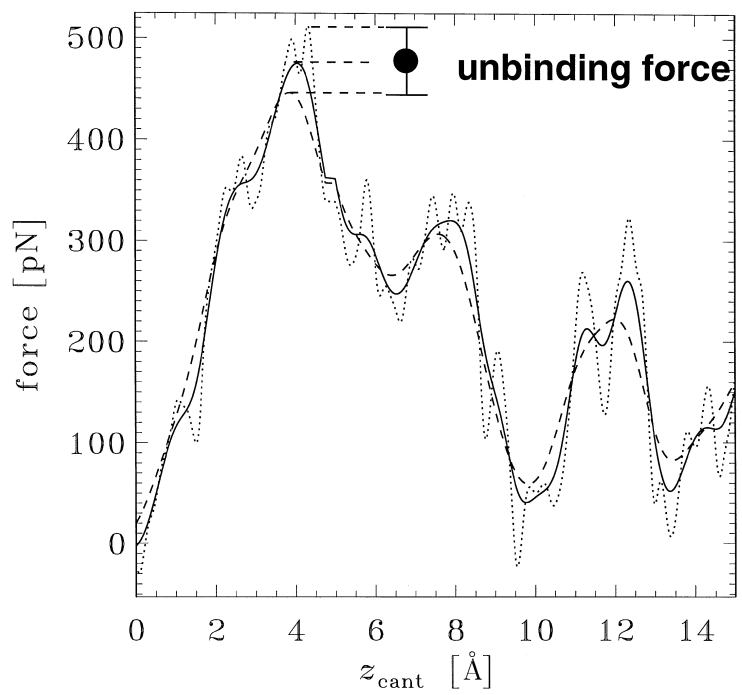

Fig. 2. Pulling force exerted on the hapten molecule during unbinding as a function of cantilever position $z_{\text {cant }}$. To eliminate thermal noise, the pulling force has been smoothed with a Gaussian filter of half widths $0.3 \AA$ (solid line), $0.1 \AA$ (dotted line) and $0.5 \AA$ (dashed line), respectively. The force maximum was interpreted as the unbinding force; the variation of this force maximum with smoothing width implies an uncertainty (error bar). with smaller (dotted line) and larger (dashed line) half widths, respectively.

The maxima of the force profile could be related to elementary rupture events like the breakage of hydrogen bonds or van der Waals contacts between the hapten and binding pocket residues. Whereas the global shape of the individual force profiles was found to be similar and, in particular, the position of pronounced maxima was reproduced in most AFM simulations, closer inspection of the force profiles revealed considerable variations particularly for $z_{\text {cant }}$ $\geqslant 4 \AA$ (data not shown), which suggests a large structural heterogeneity in the studied unbinding process.

Fig. 3 summarizes the results of all 39 AFM simulations. Shown are the computed unbinding forces (filled circles) as a function of pulling velocity. Apparently, the unbinding forces increase with pulling velocity. The linear plot (inset) focuses at large pulling velocities and demonstrates the linear increase of unbinding forces with pulling velocity that is expected from the dominating friction within that regime. In contrast, for smaller velocities the unbinding forces fall below that linear behavior. As can be seen from the main figure, which displays the same data at a logarithmic velocity scale, that deviation can be described well by considering activated processes: here, the fit of the computed unbinding forces to Eqs. (2) and (7) (solid line) shows that the deviation from the linear behavior (dashed-dotted line) is consistent with our simple extrapolation approach. The significant scatter of the computed unbinding forces as well as their individual error bars imply an uncertainty of the fit which we estimate as $\pm 15 \mathrm{pN}$ for the millisecond timescale.

An additional uncertainty of extrapolated unbinding forces is introduced by the inaccurate value for the spontaneous dissociation rate $k_{0}$. The dashed lines in the figure indicate that uncertainty, referring to $k_{0}=20 / \mathrm{s}$ (upper line) and $k_{0}=500 / \mathrm{s}$ (lower line), respectively. From the fit to $k_{0}=110 / \mathrm{s}$ (solid line) reliable values for both, $L$ and $\Delta L$, could not be obtained. We therefore assumed $\Delta L=3.6 \AA$ and obtained from the fit $L=2.2 \AA$.

Fig. 4 displays the unbinding lengths (filled circles) obtained from the individual MD AFM simulations as a function of pulling velocity. For velocities below $15 \mathrm{~m} / \mathrm{s}$ - i.e., all simulations except those 


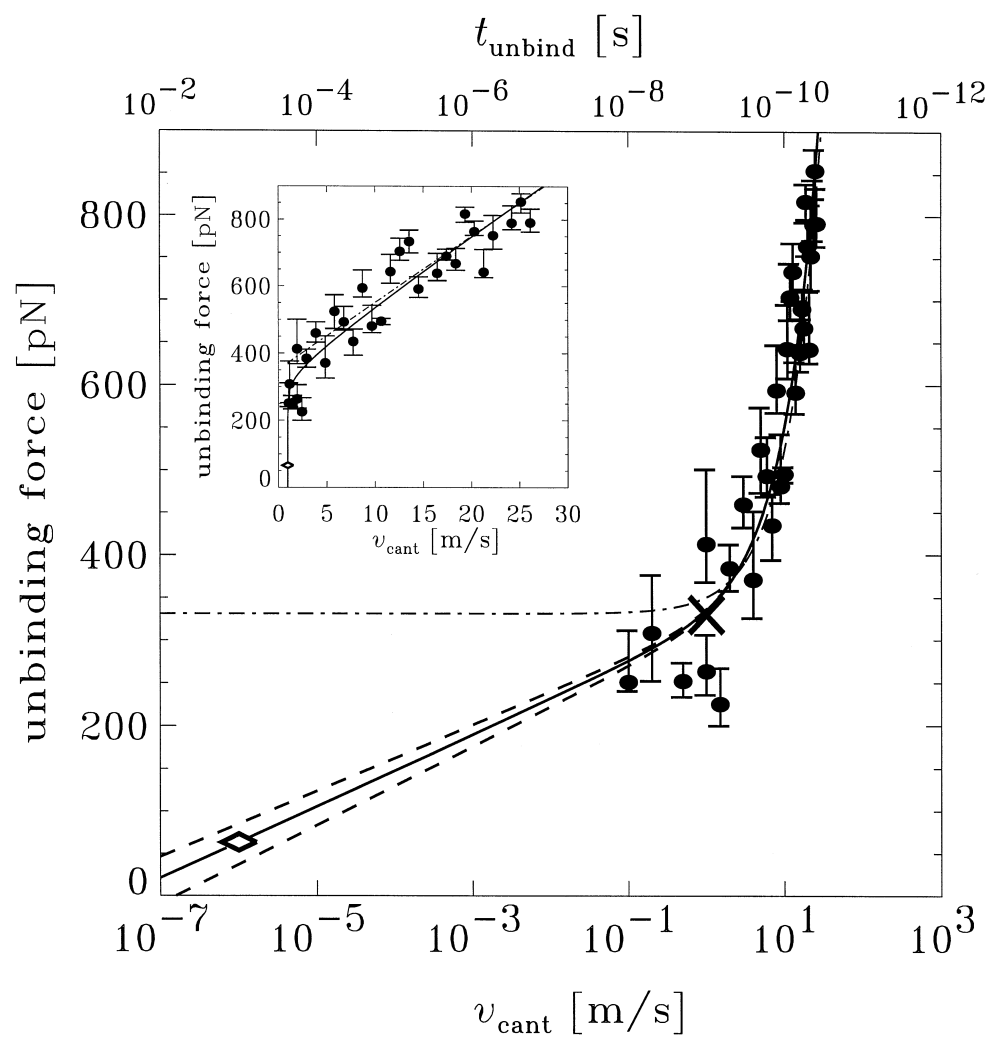

Fig. 3. Computed unbinding forces (filled circles) and predicted experimental unbinding force (diamond) as a function of pulling velocity (logarithmic scale). The crossmark indicates $v_{\text {cant }}^{*}$ and $F^{*}$, respectively. Forces above $900 \mathrm{pN}$ are not shown. The solid line shows the best fit of Eqs. (2) and (7) to the computed unbinding forces for $k_{0}=110 / \mathrm{s}$; the dashed lines depict fits for $k_{0}=20 / \mathrm{s}$ (upper line) and $k_{0}=500 / \mathrm{s}$ (lower line), respectively. The dashed-dotted line shows a fit based on friction only (i.e., $k_{0}=0$ ). The inset shows the same data at a linear velocity scale.

with extremely fast enforced unbinding - the average unbinding length (dashed line) is in good agreement with the value $L=2.2 \AA$ obtained independently from the fit. In addition, our choice of $\Delta L$ (shown as dotted lines) agrees with the simulation data in that most computed unbinding forces fall within that interval, as required by our definition of $\Delta T_{\text {trans }}$ and $\Delta L$, respectively.

Comparison of the solid, dashed, and dasheddotted fits in Fig. 3 supports the assumption that for $v_{\text {cant }}=1 \mathrm{~m} / \mathrm{s}$ (crossmark) the unbinding forces derived from the fits are essentially independent of $k_{0}$. Thus, the convenient expression for the rupture force within the activated regime, Eq. (9), is valid, and the fit yields $F^{*}=330 \mathrm{pN}$ and $\alpha_{v}^{*}=\ln \left(4.5 / \mathrm{ns} \cdot k_{0}^{-1}\right)$. For $k_{0}=110 / \mathrm{s}$ and $\alpha_{v}^{\exp }=2.7$ (i.e., $k_{v}^{\exp }=1000 / \mathrm{s}$ ) one obtains, e.g., $F_{\text {unbind }}=60 \pm 30 \mathrm{pN}$.
Detailed inspection of the computed unbinding events revealed that the scatter of the unbinding forces is caused by a surprisingly large structural heterogeneity of the unbinding pathways. After the first unbinding steps, which are characterized by the breakage of a hydrogen bond between the TYR-33L hydroxyl group and the amino group adjacent to the 2,2,6,6-tetramethyl-I-piperindinyloxy group of the hapten and the partial release of the hapten from a sandwich configuration formed by TRP-90 L and TRP-100 $\mathrm{H}$, the interactions between the hapten and the surrounding residues near the binding pocket entry differed significantly from each other. Besides these variations between the sequences of formation and rupture of local interactions, also significant structural heterogeneity was found for the unbinding pathways. A detailed analysis of that structural het- 


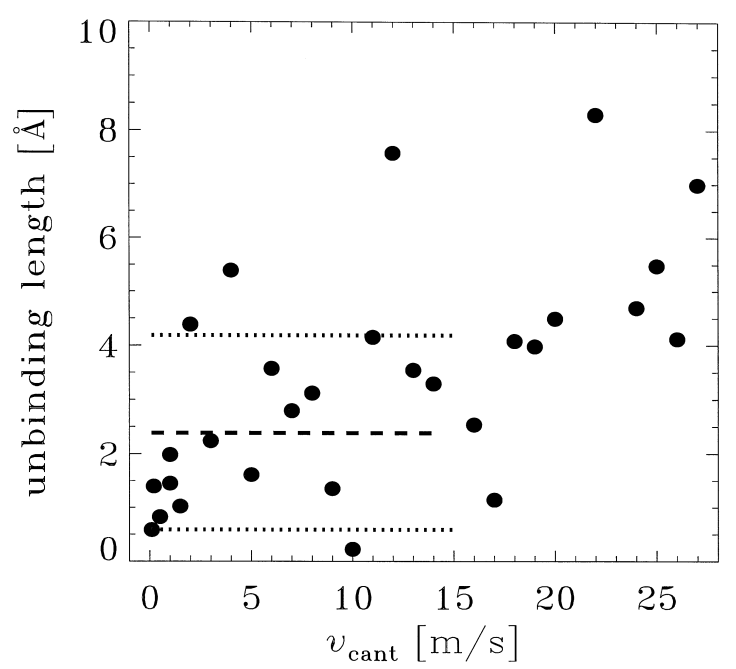

Fig. 4. Unbinding lengths (filled circles) derived from individual AFM MD simulations as a function of pulling velocity $v_{\text {cant }}$. For velocities below $15 \mathrm{~m} / \mathrm{s}$ the average unbinding length is shown as a dashed line; our choice of the unbinding length scatter, $\Delta L$, is shown as dotted lines.

erogeneity of unbinding pathways will be given elsewhere.

\section{Discussion}

A series of extended MD AFM simulations of the solvated AN02/DNP-hapten complex was performed and a simple analytical model was presented that allows extrapolation of the computed unbinding forces from the MD timescale to the timescale at which single molecule AFM experiments are typically carried out. As time-dependent non-equilibrium effects our model considers friction and activated processes, which modify the unbinding force as a function of the timescale at which unbinding is enforced. As additional information the experimental value for the spontaneous dissociation rate of the complex is used.

It has been asserted that AFM MD simulations were generally restricted to the friction regime, which would render extrapolations into the activated regime - which is relevant to AFM experiments - problematic, if not impossible [17]. The extended simulations presented here cover both: one order of magnitude of the activated regime, and nearly two orders of magnitude of the friction regime. In the case of the AN02/DNP complex that was subject of the present study, activated processes appear to dominate for pulling velocities smaller than $1 \mathrm{~m} / \mathrm{s}$. In particular, the force contribution of the friction in the MD AFM simulation with smallest pulling velocity $(0.1 \mathrm{~m} / \mathrm{s})$ was below $3 \mathrm{pN}$, whereas at that timescale activated processes lowered the unbinding force by nearly 100 $\mathrm{pN}$, which clearly demonstrates the dominance of activated processes within the respective regime. For pulling velocities as large as $25 \mathrm{~m} / \mathrm{s}$ the corresponding values are $500 \mathrm{pN}$ for friction and a vanishing contribution due to activated processes, respectively, thus demonstrating the dominance of frictional forces in that regime.

We note that already in Ref. [15] a variation of unbinding force with pulling velocity for the streptavidin-biotin complex was observed. There, as a first approximation, the linear behavior apparent in our simulations was extrapolated linearly to the experimental timescale and good agreement with experiment was obtained. This simple approach has been criticized [17] since it would not take into account activated processes, which, as is also demonstrated in the present work, lower unbinding forces at long timescales. Accordingly it was argued that, by considering only friction as done in Ref. [15], the unbinding force at the AFM timescale was overestimated dramatically. However, our present analysis strongly supports our initial assumption that in the streptavidin-biotin case activated processes lower the unbinding force at the AFM timescale only slightly. In fact, using the very low streptavidinbiotin spontaneous dissociation rate of $k_{0} \approx(3$ days $)^{-1}$, we now estimate a reduction by less than $70 \mathrm{pN}$ as compared to the value of $550 \mathrm{pN}$ claimed in Ref. [17] on the basis of a diffusion coefficient derived from Mößbauer data for the heme group in myoglobin.

For the AN02/DNP system studied in the present Letter the considerably higher spontaneous dissociation rate of the AN02-hapten complex required consideration of activated processes; their neglect would indeed imply an unbinding force that is larger by a factor of four than the value of $60 \pm 30 \mathrm{pN}$ suggested here for the millisecond timescale. 


\section{Acknowledgements}

This work was supported by the Deutsche Forschungsgemeinschaft, Grant GR 1590/1-2.

\section{References}

[1] E.A. Padlan, Mol. Immunol. 31 (1994) 169.

[2] S. Dickman, Science 280 (1998) 1196.

[3] O. Kristensen, D.G. Vassylyev, F. Tanaka, K. Morikawa, I. Fujii, J. Mol. Biol. 281 (1998) 501.

[4] G.U. Lee, D.A. Kidwell, R.J. Colton, Langmuir 10 (1994) 354.

[5] E.-L. Florin, V.T. Moy, H.E. Gaub, Science 264 (1994) 415.

[6] M. Rief, M. Gautel, F. Oesterhelt, J.M. Fernandez, H.E. Gaub, Science 276 (1997) 1109.

[7] M.S.Z. Kellermayer, S.B. Smith, H.L. Granzier, C. Bustamante, Science 276 (1997) 1112.

[8] L. Tskhovrebova, J. Trinck, J.A. Sleep, R.M. Simmons, Nature 387 (1997) 308.

[9] G.U. Lee, L.A. Chrisey, R.J. Colton, Science 266 (1994) 771.

[10] M. Rief, F. Oesterhelt, B. Heymann, H.E. Gaub, Science 275 (1997) 1295.

[11] P. Hinterdorfer, W. Baumgartner, H.J. Gruber, K. Schlicher, H. Schindler, Proc. Natl. Acad. Sci. USA 93 (1996) 3477.

[12] U. Dammer, M. Hegner, D. Anselmetti, P. Wagner, M. Dreier, W. Huber, H.-J. Güntherodt, Biophys. J. 70 (1996) 2437.

[13] S. Allen, X. Chen, J. Davies, M.C. Davies, A.C. Dawkes, J.C. Edwards, C.J. Roberts, J. Sefton, S.J.B. Tendler, P.M. Williams, Biochemistry 36 (1997) 7457.

[14] R. Ros, F. Schwesinger, D. Anselmetti, M. Kubon, R. Schäfer, A. Plückthun, L. Tiefenauer, Proc. Natl. Acad. Sci. USA 95 (1998) 7402.

[15] H. Grubmüller, B. Heymann, P. Tavan, Science 271 (1996) 997.
[16] M.W. Konrad, J.I. Bolonick, J. Am. Chem. Soc. 118 (1996) 10986.

[17] S. Izrailev, S. Stepaniants, M. Balsera, Y. Oono, K. Schulten, Biophys. J. 72 (1997) 1568.

[18] S. Marrink, O. Berger, P. Tieleman, F. Jähnig, Biophys. J. 74 (1998) 931.

[19] H. Lu, B. Isralewitz, A. Krammer, V. Vogel, K. Schulten, Biophys. J. 75 (1998) 662.

[20] W.F. van Gunsteren, H.J.C. Berendsen, Angew. Chem. Int. Ed. Engl. 29 (1990) 992.

[21] A.T. Brünger, D.J. Leahy, T.R. Hynes, R.O. Fox, J. Mol. Biol. 221 (1991) 239.

[22] E. Evans, K. Ritchie, Biophys. J. 72 (1997) 1541.

[23] F.C. Bernstein, T.F. Koetzle, G.J.B. Williams, E.F. Meyer, M.D. Brice, J.R. Rodgers, O. Kennard, T. Shimanouchi, M.J. Tasumi, J. Mol. Biol. 112 (1977) 535.

[24] M. Eichinger, H. Grubmüller, H. Heller, User Manual for EGO VIII, Release 2.0, electronic access: http:// www.mpibpc.gwdg.de/abteilungen/071/ego.html.

[25] A. Brünger, X-PLOR, version 3.1 edn., The Howard Hughes Medical Institute and Department of Molecular Biophysics and Biochemistry, Yale University, New Haven, CT, 1992.

[26] M. Eichinger, H. Grubmüller, H. Heller, P. Tavan, J. Comput. Chem. 18 (1997) 1729.

[27] UniChem 3.0 Manual, Cray Research, 655 Lone Oak Drive, Eagan, MN 55151, 1997.

[28] W.F. van Gunsteren, H.J.C. Berendsen, Mol. Phys. 34 (1977) 1311.

[29] W.L. Jorgensen, J. Chandrasekhar, J.D. Madura, R.W. Impey, M.L. Klein, J. Phys. Chem. 79 (1983) 926.

[30] H. Grubmüller, Solvate: A Program to Create Atomic Solvent Models, 1996 (electronic publication, http://www.mpibpc. gwdg.de/abteilungen/071/hgrub/solvate/docu.html).

[31] C.L. Brooks III, M. Karplus, J. Chem. Phys. 79 (1983) 6312.

[32] A. Brünger, C.L. Brooks III, M. Karplus, Chem. Phys. Lett. 105 (1984) 495.

[33] R. Kossmann, Master's Thesis, Ludwig-Maximilians-Universität München, 1997.

[34] M. Rief, M. Gautel, A. Schemmel, H. Gaub, Biophys. J. 75 (1998) 3008.

[35] M. Martinez-Yamout, H.M. McConnell, J. Mol. Biol. 244 (1994) 301. 